Supplement 1: List of radiolarian taxa included in the SO-RAD dataset.

\section{Taxon name}

Acanthodesmia micropora (Popofsky)

Acanthodesmia viniculata (Müeller)

Acanthosphaera Ehrenberg spp.

Acrobotrys teralans Renz

Acrosphaera arktios (Nigrini)

Acrosphaera cyrtodon (Haeckel)

Acrosphaera inflata Haeckel

Acrosphaera lappacea (Haeckel)

Acrosphaera mercurius Lazarus

Acrosphaera murrayana (Haeckel)

Acrosphaera spinosa Caulet

Actinomma antarcticum (Haeckel)

Actinomma arcadophorum Haeckel

Actinomma boreale Cleve

Actinomma delicatulum (Dogiel and Reschetnjak)

Actinomma Haeckel

Actinomma hastatum (Haeckel)

Actinomma leptodermum (Jørgensen) gr.

Actinomma medianum Nigrini

Actinomma popofski (Petrushevskaya)

Actinomma sol Cleve

Actinomma trinacria (Haeckel)

Actinosphaera Hollande and Enjumet

Amphiplecta acrostoma Haeckel

Amphirhopalum ypsilon Haeckel

Androspyris fenestrata (Haeckel)

Anomalacantha dentata Mast

Antarctissa denticulata (Ehrenberg)

Antarctissa Petrushevskaya spp.

Antarctissa strelkovi Petrushevskaya

Anthocyrtidium ophirense (Ehrenberg)

Anthocyrtidium zanguebaricum (Ehrenberg)

Arachnocorys umbellifera Haeckel

Archipilium orthopterum Haeckel

Artobotrys borealis (Cleve)

Artostrobus annulatus (Bailey)

Artostrobus joergenseni Petrushevskaya gr.

Axoprunum stauraxonium Haeckel

Botryocampe inflata (Bailey)

Botryocephalina armata Petrushevskaya

Botryocyrtis scutum (Harting)

Botryopyle cribrosa (Ehrenberg)

Botryostrobus aquilonaris (Bailey)

Botryostrobus auritus/australis (Ehrenberg)

Botryostrobus Haeckel

\section{Reference}

Petrushevskaya, 1971

Petrushevskaya, 1971

Boltovskoy, 1998; Boltovskoy and Riedel, 1980

Renz, 1976

Nigrini, 1970

Strelkov and Reshetnyak, 1971

Haeckel, 1887

Johnson and Nigrini, 1980

Lazarus, 1992

Hilmers, 1906

Caulet, 1986

Nigrini and Moore, 1979

Haeckel, 1887

Cortese and Bjørklund, 1998

Petrushevskaya, 1967

Haeckel, 1860

Rogers and De Deckker, 2007

Boltovskoy, 1998; Cortese and Bjørklund, 1998

Nigrini and Moore, 1979

Petrushevskaya, 1967

Boltovskoy, 1998

Cortese and Bjorklund, 1998

Hollande and Enjumet, 1960

Haeckel, 1887

Haeckel, 1887

Goll, 1980

Mast, 1910

Petrushevskaya, 1967

Nigrini and Moore, 1979; Petrushevskaya, 1967

Petrushevskaya, 1967

Petrushevskaya, 1968

Haeckel, 1887

Haeckel, 1860

Haeckel, 1887

Petrushevskaya, 1971

Boltovskoy, 1998; Petrushevskaya, 1967, 1971

Petrushevskaya, 1967

Nigrini and Moore, 1979

Matsuzaki et al., 2015; Itaki, 2009; Haeckel, 1887

Petrushevskaya, 1965

Boltovskoy, 1998; Nigrini and Moore, 1979

Haeckel, 1887

Boltovskoy, 1998; Nigrini and Moore, 1979; Petrushevskaya, 1967

Boltovskoy, 1998; Nigrini and Moore, 1979; Petrushevskaya, 1967

Haeckel, 1887 
Buccinosphaera invaginata Haeckel

Callimitra Haeckel

Carpocanarium papillosum (Ehrenberg)

Carpocanistrum Haeckel spp.

Cenosphaera cristata Haeckel

Cenosphaera Ehrenberg spp.

Cenosphaera sp. aff. C. perforata Haeckel

Ceratocyrtis galeus (Cleve)

Ceratocyrtis histricosa (Joergensen)

Chromyechinus antarctica (Dreyer)

Circodiscus microporus (Stoehr)

Cladococcus abietinus Haeckel

Cladoscenium ancoratum Haeckel

Cladoscenium limbatum Joergensen

Cladoscenium sp. 1

Cladoscenium tricolpium (Haeckel)

Clathrocanium coarctatum Ehrenberg

Collosphaera confossa Takahashi

Collosphaera huxleyi Mueller

Collosphaera macropora Popofsky

Collosphaera tuberosa Haeckel

Collosphaeridae

Cornutella profunda Ehrenberg

Corocalyptra craspedota (Jørgensen)/cervus

(Ehrenberg)

Corythospyris stapedius (Haeckel)

Cubotholus Haeckel

Cycladophora bicornis (Popofsky)

Cycladophora davisiana cornutoides

(Petrushevskaya)

Cycladophora davisiana davisiana (Ehrenberg)

Cypassis Haeckel

Cypassis irregularis Nigrini

Cyrtolagena laguncula (Haeckel)

Dendrospyris Haeckel

Dictyocoryne profunda Ehrenberg

Dictyocoryne truncatum (Ehrenberg)

Dictyophimus archipilium Petrushevskaya

Dictyophimus crisiae Ehrenberg/hirundo

(Haeckel)

Dictyophimus Ehrenberg

Dictyophimus infabricatus Nigrini

Dictyophimus killmari (Renz)

Dictyophimus macropterus (Ehrenberg)

Didymocyrtis tetrathalamus (Haeckel)

Druppatractus irregularis Popofksy

Druppatractus variabilis Dumitrica

Enneaphormis rotula Haeckel

Euchitonia elegans (Ehrenberg)/furcata Ehrenberg gr.
Haeckel, 1887

Haeckel 1881

(Ehrenberg), 1872

Boltovskoy, 1998; Nigrini and Moore, 1979

Haeckel, 1887

Boltovskoy, 1998; Nigrini and Moore, 1979

Benson, 1983

Bjoerklund, 1976

Petrushevskaya, 1971

Itaki, 2009; Petrushevskaya, 1967

Petrushevskaya and Kozlova, 1972

Haeckel, 1887

Haeckel, 1887

Joergensen, 1905

No reference

Joergensen, 1900

Ehrenberg, 1872

Takahashi, 1991

Mueller, 1855

Popofsky, 1917

Haeckel, 1887

Several species of the subfamily Collosphaeridae Mueller;

Boltovskoy, 1998

Boltovskoy, 1998

Bjørklund, 1976; Boltovskoy, 1998; Petrushevskaya, 1971

Goll, 1978

Haeckel 1887

Nigrini and Moore, 1979

Petrushevskaya, 1967

Boltovskoy, 1998; Nigrini and Moore, 1979; Petrushevskaya, 1967

Haeckel 1887

Nigrini, 1968

Boltovskoy, 1998; Petrushevskaya, 1971

Haeckel 1881

Boltovskoy, 1998; Nigrini and Moore, 1979

Boltovskoy, 1998; Nigrini and Moore, 1979

Petrushevskaya , 1975

Boltovskoy, 1998; Nigrini and Moore, 1979

Ehrenberg 1847

Nigrini, 1968

(Renz), 1974

Takahashi, 1991

Boltovskoy, 1998; Nigrini and Moore, 1979

Popofsky, 1912

Dumitrica, 1973; Takahashi, 1991

Haeckel, 1887

Boltovskoy, 1998 
Eucyrtidium acuminatum (Ehrenberg)

Eucyrtidium annulatum (Popofsky)

Eucyrtidium anomalum (Haeckel)

Eucyrtidium calvertense (Martin)

Eucyrtidium cienkowskii Haeckel

Eucyrtidium Ehrenberg emend. Nigrini spp.

Eucyrtidium hexagonatum Haeckel

Eucyrtidium hexastichum Haeckel

Eucyrtidium octocolum (Haeckel)

Eucyrtidium teuscheri Haeckel

Euphysetta elegans Borgert

Euphysetta nathorstii Cleve

Euphysetta staurocodon Haeckel

Gondwanaria campanulaeformis (Campbell and Clark)

Gondwanaria dogieli Petrushevskaya

Gondwanaria Petrushevskaya and Kozlova

Gonosphaera primordialis Jørgensen 1905

Heliodiscus asteriscus Haeckel

Hexacontium arachnoidale Hollande

Hexacontium enthacanthum Joergensen

Hexacontium gigantheum Cortese and Bjorklund

Hexacontium laevigatum Haeckel

Hexacontium melpomene (Haeckel)

Hexacontium pachydermum Joergensen

Hexalonche amphisiphon Haeckel

Hexapyle dodecantha Haeckel gr.

Hexastylus spp. Haeckel gr.

Hymeniastrum euclidis Haeckel

Lamprocyclas maritalis Haeckel s.l.

Lamprocyrtis hannai Campbell and Clark

Lamprocyrtis nigriniae (Caulet)

Lampromitra coronata Haeckel

Lampromitra quadricuspis Haeckel

Lampromitra schultzei (Haeckel)

Larcopyle buetschlii Dreyer

Larcopyle polyacantha (Campbell and Clark)

Larcopyle pylomaticus (Riedel)

Larcopyle weddellium Lazarus et al.

Larcospira bulbosa Goll and Bjoerklund

Larcospira quadrangula Haeckel

Larnacilla typus Haeckel

Lipmanella bombus Haeckel

Lipmanella dictyoceras (Haeckel)

Lirella melo (Cleve)

Liriospyris reticulata (Ehrenberg)

Litharachnium tentorium Haeckel

Litheliidae/Pyloniidae Haeckel

Lithelius minor Jørgensen gr.
Ehrenberg, 1847

(Popofsky), 1913

(Haeckel) Haeckel, 1862

Martin, 1904

Haeckel, 1887

Boltovskoy, 1998; Nigrini and Moore, 1979

Haeckel, 1887

Haeckel, 1887

Petrushevskaya, 1971

Haeckel, 1887

Borgert, 1902

Cleve, 1899

Takahashi, 1991

(Campbell and Clark), 1945

Petrushevskaya, 1979

Petrushevskaya and Kozlova 1975

Jørgensen 1905

Boltovskoy, 1998; Nigrini and Moore, 1979

Hollande, 1960

Joergensen, 1900

Cortese and Bjorklund, 1998

Haeckel, 1887

Van de Paverd, 1995

Joergensen, 1900

Haeckel, 1887

Boltovskoy, 1998; Nigrini and Moore, 1979

Rogers and De Deckker, 2007

Nigrini and Moore, 1979

Boltovskoy, 1998; Nigrini and Moore, 1979

Boltovskoy, 1998

Kling, 1973

Haeckel, 1887

Haeckel, 1887

Takahashi, 1991

Boltovskoy, 1998; Nigrini and Moore, 1979

Lazarus et al., 2005

Lazarus et al., 2005

Lazarus et al., 2005

Goll and Bjoerklund, 1989

Haeckel, 1887

Haeckel, 1887; Dumitrica, 1989 (as Larnacopylomma)

Haeckel, 1887

Kling, 1973

Takahashi, 1991

Goll, 1968

Haeckel, 1860

Haeckel, 1887

Boltovskoy, 1998; Nigrini and Moore, 1979 
Lithelius nautiloides Popofsky

Lithelius sp. 1

Lithelius spiralis Haeckel

Lithocampe ? furcaspiculata (Harting)

Lithocampe ? platycephala (Ehrenberg)

Lithomelissa (?) sp. A Petrushevskaya

Lithomelissa Ehrenberg

Lithomelissa hystrix Joergensen

Lithomelissa laticeps Joergensen

Lithomelissa setosa Jørgensen

Lithomelissa sp. B

Lithomelissa thoracites Haeckel

Lithostrobus cuspidatus Haeckel

Lonchosphaera spicata Popofsky

Lophophaena buetschlii (Haeckel)

Lophophaena Ehrenberg

Lophophaena hispida (Ehrenberg)

Lophospyris pentagona (Ehrenberg)

Mitrocalpis araneafera Popofsky

Peridium longispinum Jørgensen

Peripyramis circumtexta Haeckel

Peromelissa phalacra Haeckel

Phormacantha hystrix (Joergensen)

Phormospyris stabilis Goll

Phormostichoartus corbula (Harting)

Phorticium clevei Haeckel

Plectacantha cremastoplegma Nigrini

Plectacantha oikiskos Jørgensen

Plectopyramis dodecomma Haeckel

Porodiscus Haeckel

Protocystis balfouri (Murray)

Protocystis harstoni (Murray)

Protocystis swirei (Murray)

Pseudocubus obeliscus Haeckel

Pseudodictyophimus bicornis (Ehrenberg)

Pseudodictyophimus gracilipes (Bailey) s.l.

Pseudodictyophimus gracilipes multispinus

Bernstein

Pseudodictyophimus horrida (Petrushevskaya)

Pseudodictyophimus platycephalus (Haeckel)

Pterocanium auritum Nigrini \& Caulet

Pterocanium charybdeum (Mueller)

Pterocanium korotnevi (Dogel)

Pterocanium praetextum eucolpum Haeckel

Pterocanium praetextum praetextum (Ehrenberg)

Pterocorys clausus (Popofsky)/zancleus (Mueller)

Pterocorys hertwigii (Haeckel)

Pterocorys minythorax (Nigrini)
Boltovskoy, 1998; Nigrini and Moore, 1979; Petrushevskaya, 1967

No reference

Haeckel, 1860

Boltovskoy, 1998; Nigrini and Moore, 1979 (as P. corbula);

Petrushevskaya, 1967

Cortese et al., 2003; Petrushevskaya, 1967

Petrushevskaya, 1967

Ehrenberg, 1847

Joergensen, 1900

Joergensen, 1905

Cortese et al., 2003; Takahashi, 1991

No reference

Petrushevskaya, 1971

Haeckel 1887

Popofsky, 1908

Petrushevskaya, 1971

Ehrenberg 1847

Petrushevskaya, 1971

(Ehrenberg), 1872; Petrushevskaya, 1971

Popofsky, 1908

Bjørklund, 1976; Bjørklund et al., 1998

Haeckel, 1887

Boltovskoy, 1998; Petrushevskaya, 1971

Joergensen, 1905

Goll, 1976

Nigrini, 1977

Boltovskoy, 1998; Petrushevskaya, 1967

Nigrini, 1968

Jørgensen, 1905

Haeckel, 1887

Haeckel, 1881

Borgert, 1911

Murray, 1885; Schroeder, 1913

Haecker, 1908

Haeckel, 1887

Welling, 1997

Boltovskoy, 1998 (as Dictyophimus gracilipes)

Bernstein, 1934

Petrushevskaya, 1975

Petrushevskaya, 1971

Nigrini and Caulet, 1992

(Mueller), 1858

Dogel and Reshetnyak , 1952

Haeckel, 1887

Boltovskoy, 1998; Nigrini and Moore, 1979; Petrushevskaya, 1971

Boltovskoy, 1998; Nigrini and Moore, 1979

Petrushevskaya, 1971

Nigrini and Moore, 1979 
Pteroscenium pinnatum Haeckel

Pylospira octopyle Haeckel

Rhizoplegma boreale Popofsky

Saccospyris antarctica Haecker

Saccospyris conithorax Petrushevskaya

Saccospyris spp. Haecker

Saturnalis circularis Haeckel

Schizodiscus stylotrochoides Dogiel

Sethoconus tabulatus Haeckel

Siphocampe arachnea (Ehrenberg) gr.

Siphocampe lineata (Ehrenberg) gr.

Siphonosphaera socialis Haeckel

Solenosphaera zanguebarica Brandt

Sphaeropyle robusta Kling

Sphaerozoum punctatum Müller

Spongaster tetras Ehrenberg

Spongodiscus resurgens Ehrenberg

Spongopyle osculosa Dreyer

Spongotrochus glacialis Popofsky

Spongotrochus Haeckel/Spongopyle Dreyer

Spongurus cylindricus Haeckel

Spongurus sp. cf. S. elliptica (Ehrenberg)

Stichocorys seriata Joergensen

Stichopilidium kruegeri (Popofsky)

Stichopilium bicorne Haeckel

Stylatractus Haeckel

Stylatractus neptunus Haeckel

Stylatractus ostracion (Haeckel)

Stylatractus sp. A

Stylochlamydium asteriscus Haeckel

Stylochlamydium Haeckel

Stylochlamydium venustum (Bailey)

Stylodictya aculeata Jørgensen

Stylodictya Ehrenberg

Stylodictya tenuispina Jørgensen

Stylodictya validispina Haeckel

Stylosphaera Ehrenberg

Styptosphaera ? spumacea Haeckel

Tessarastrum straussii Haeckel

Tetraphormis dodecaster (Haeckel)

Tetrapyle octacantha Mueller/Octopyle stenozona

Haeckel gr.

Theocorys veneris Haeckel

Theocorythium trachelium (Ehrenberg) s.1.

Tholospyris gephyristes Huelsemann

Triceraspyris antarctica (Haecker)

Trissocyclidae Haeckel, emend. Goll

Trisulcus testudus Petrushevskaya

Trisulcus triacanthus Popofsky
Haeckel, 1887

Haeckel, 1887

Popofsky, 1908

Boltovskoy, 1998; Petrushevskaya, 1967

Petrushevskaya, 1965

Haecker, 1907

Haeckel, 1887

Petrushevskaya, 1967

Haeckel, 1887

Nigrini, 1977

Takahashi, 1991

Haeckel, 1887

Brandt, 1905

Kling, 1973

Rogers and De Deckker, 2007

Boltovskoy, 1998; Nigrini and Moore, 1979

Boltovskoy, 1998

Nigrini and Moore, 1979

Boltovskoy, 1998; Nigrini and Moore, 1979; Petrushevskaya, 1967

Haeckel, 1860; Dreyer, 1889

Haeckel, 1860

(Ehrenberg), 1872

Joergensen, 1905

Nishimura \& Yamauchi, 1984

Haeckel, 1887

Haeckel, 1887

Nigrini and Moore, 1979; Petrushevskaya, 1967

Haeckel, 1887

No reference

Boltovskoy, 1998; Nigrini and Moore, 1979

Haeckel 1881

Boltovskoy, 1998; Nigrini and Moore, 1979

Boltovskoy, 1998; Nigrini and Moore, 1979; Petrushevskaya, 1967

Ehrenberg, 1847

Nigrini and Moore, 1979

Boltovskoy, 1998; Nigrini and Moore, 1979; Petrushevskaya, 1967

Ehrenberg, 1847

Nigrini and Moore, 1979

Haeckel, 1887; Takahashi, 1991 (as Amphirhopalum straussii)

Takahashi, 1991

Boltovskoy, 1998; Nigrini and Moore, 1979

Haeckel, 1887

Boltovskoy, 1998; Nigrini and Moore, 1979; Petrushevskaya, 1971

Bjørklund et al., 1998

Haecker, 1908

Haeckel, 1881; Goll, 1968

Petrushevskaya, 1971

Popofsky, 1913 
Zygocircus productus (Hertwig)

Nassellaria Ehrenberg, indet.

Spumellaria Ehrenberg, indet.
Haeckel, 1887

Ehrenberg, 1875

Ehrenberg, 1875 
Supplement 2: Recommended taxonomic groupings to simplify/harmonise the dataset. Adapted from Cortese \& Prebble, 2015.

\begin{tabular}{|c|c|}
\hline Taxa & Group \\
\hline Actinomma delicatulum & \multirow{2}{*}{ ACTINOMMA DELICATULUM/POPOFSKII } \\
\hline Actinomma popofskii & \\
\hline Anthocyrtidium ophirense & \multirow{2}{*}{ ANTHOCYRTIDIUM OPHIRENSE/ZANGUEBARICUM } \\
\hline Anthocyrtidium zanguebaricum & \\
\hline Acrosphaera arktios & \multirow{14}{*}{ COLLOSPHAERIDAE } \\
\hline Acrosphaera cyrtodon & \\
\hline Acrosphaera inflata & \\
\hline Acrosphaera lappacea & \\
\hline Acrosphaera murrayana & \\
\hline Acrosphaera spinosa & \\
\hline Buccinosphaera invaginata & \\
\hline Collosphaerid & \\
\hline Collosphaera confossa & \\
\hline Collosphaera huxleyi & \\
\hline Collosphaera macropora & \\
\hline Collosphaera tuberosa & \\
\hline Solenosphaera zanguebarica & \\
\hline Siphonosphaera socialis & \\
\hline Druppatractus variabilis & \multirow{2}{*}{ DRUPPATRACTUS VARIABILIS/OSTRACION } \\
\hline Stylatractus ostracion & \\
\hline Eucyrtidium spp. & \multirow{10}{*}{ EUCYRTIDIUM SPP } \\
\hline Eucyrtidium acuminatum & \\
\hline Eucyrtidium annulatum & \\
\hline Eucyrtidium anomalum & \\
\hline Eucyrtidium calvertense & \\
\hline Eucyrtidium cienkowskii & \\
\hline Eucyrtidium hexagonatum & \\
\hline Eucyrtidium hexastichus & \\
\hline Eucyrtidium octocolum & \\
\hline Eucyrtidium teuscheri & \\
\hline Hexacontium enthacanthum & \multirow{2}{*}{ HEXACONTIUM PACHY/ENTHA } \\
\hline Hexacontium pachydermum & \\
\hline Lampromitra coronata & \multirow{2}{*}{ LAMPROMITRA PENTALACTIS/QUADRICUSPIS } \\
\hline Lampromitra quadricuspis & \\
\hline Lithelius minor & \multirow{2}{*}{ LITHELIUS MINOR/SPIRALIS } \\
\hline Lithelius spiralis & \\
\hline Lithomelissa setosa & \multirow{3}{*}{ LITHOMELISSA SETOSA Group } \\
\hline Lithomelissa hystrix & \\
\hline Trisulcus testudus & \\
\hline Phormacantha hystrix & \multirow{3}{*}{ PHORMACANTHA/PLECTACANTHA } \\
\hline Plectacantha cremastoplegma & \\
\hline Plectacantha oikiskos & \\
\hline Pseudodictyophimus gracilipes & \multirow{4}{*}{ PSEUDODICTYOPHIMUS GRACILIPES S.L. } \\
\hline Pseudodictyophimus gracilipes multispinus & \\
\hline Pseudodictyophimus platycephalus & \\
\hline Pseudodictyophimus bicornis & \\
\hline Pterocanium praetextum praetextum & \multirow{2}{*}{ PTEROCANIUM PRAETEXTUM/EUCOLPUM } \\
\hline Pterocanium praetextum eucolpum & \\
\hline Saccospyris antarctica & \multirow{2}{*}{ SACCOSPYRIS ANTARCTICA } \\
\hline Saccospyris conythorax & \\
\hline Spongopyle osculosa & \multirow{2}{*}{ SPONGOPYLE OSCULOSA/RESURGENS } \\
\hline Spongodiscus resurgens & \\
\hline
\end{tabular}

